\title{
Dipolar interactions and anisotropic magnetoresistance in metallic granular systems
}

\author{
J. Viana Lopes, J. M. B. Lopes dos Santos and Yu. G. Pogorelov \\ Centro de Física do Porto and Departamento de Fúsica, Faculdade de Ciências, Universidade do Porto, \\ Rua do Campo Alegre, 687, 4169-007 Porto, Portugal
}

\begin{abstract}
We revisit the theory of magnetoresistance for a system of nanoscopic magnetic granules in metallic matrix. Using a simple model for the spin dependent perturbation potential of the granules, we solve Boltzmann equation for the spin dependent components of the non equilibrium electronic distribution function. For typical values of the geometric parameters in granular systems, we find a peculiar structure of the distribution function of conduction electrons, which is at variance with the two-current model of conduction in inhomogeneous systems. Our treatment explicitly includes the effects of dipolar correlations yielding a magnetoresistance ratio which contains, in addition to the term proportional to the square of uniform magnetization $\langle\boldsymbol{\mu}\rangle$, a weak anisotropic contribution depending on the angle between electric and magnetic fields, and arising from the anisotropic character of dipolar interactions.
\end{abstract}

\section{INTRODUCTION}

Granular magnetic systems displaying giant magnetoresistance (GMR) effect [1, 2|, were put forward as an alternative to the previously known magnetic multilayers [3, 何, due to their practical advantages of easier fabrication and higher stability. On the other hand, the relevant physics in granular systems presents greater challenges than in the case of multilayered systems, for which classical [5], semiclassical [6] or quantum [7] solutions are available. Of course, in all these cases the negative GMR has the same origin in that the conduction electrons with two spin polarizations flow easier at increasing alignment of localized magnetic moments. But a difficulty results from the fact that, while the magnetic state of a multilayered system is described by only few classical variables (the orientations of magnetization in each magnetic layer), that of a granular system involves a statistical ensemble of nanoscopic granule magnetic moments which generally cannot be considered independent. Also the magnetotransport in $3 \mathrm{D}$ granular systems cannot be reduced to any of the paradigmatic cases in the layered systems, current-in-plane (CIP) or current-perpendicular-to-plane (CPP) [8]. Nevertheless, currently accepted treatments of magnetotransport in inhomogeneous materials (both granular and multilayered) share a common concept: the two-current model, in which the spin up and spin down electron subsystems carry current independently [9, 10, 11, 12]. This model reasonably reproduces the main features of GMR in granular materials. The most common one is the proportionality of the magnetoresistance ratio $\Delta \rho / \rho$ to the square of uniform magnetization, $\Delta \rho / \rho \propto m^{2}$, as first observed by Xiao et al [2] and theoretically explained by assuming the granules to have a single size and to be uncorrelated [12]. The observed proportionality of $\Delta \rho$ to the inverse of granule diameter $d[10,13]$ is accounted in these models by assuming that the spin dependent scattering in mainly at the surface of the granules. Experiments also show deviations from $m^{2}$ behavior [2, 11, 14, 15] which have been attributed to granule size dispersion
12, 14, 17], and/or correlations between the granular moments 111, 16, 17, 18, 201, most pronounced when the applied magnetic field is not too strong compared with the intergranule (dipolar) interactions.

However, despite appearances, there are some issues open for discussion in the current accounts of magnetotransport in granular materials.

The treatments referred to above usually consider the scattering from individual impurities inside the granules to be incoherent, despite the fact that electronic meanfree path can be much larger than granule diameter or even inter granule distance $[10]$. In the present paper we extend a model put forward by Pogorelov et al [17] in which the scattering was considered coherent from the whole granule volume and the geometrical factors arising from this coherence where properly taken into account, albeit within the context of two-current model. We now perform a full treatment of the kinetic Boltzmann equation and find that, within this framework, the two-current model does not hold. In the presence of dominantly forward scattering, spin flip processes become a crucial determining factor of the electronic distribution function. In this model the $1 / d$ dependence of $\Delta \rho$ is obtained without assuming specific surface scattering.

The effect of magnetic correlations on transport is usually treated in the assumption that the resistance is proportional to $\left\langle\boldsymbol{\mu}_{1} \cdot \boldsymbol{\mu}_{2}\right\rangle$ [11, 16, 18] (where $\boldsymbol{\mu}_{1}$ and $\boldsymbol{\mu}_{2}$ are the magnetic moments of granules), a phenomenological result that can be traced back to Gittleman et al [21]. The coherent scattering model of Ref. 117 put this result on a firmer footing. Treating in this model the inverse relaxation time as a spin dependent tensor, determined by a squared Born scattering amplitude of conduction electrons by magnetic granules, one readily gets a correlation term $\left\langle\boldsymbol{\mu}_{1} \cdot \boldsymbol{\mu}_{2}\right\rangle$ due to coherent spin-dependent scatterings by the moments $\boldsymbol{\mu}_{1,2}$ of two different granules. Also this theory determines the proper weighting, with distance, of the contribution of this correlation to transport. Other known approaches to magnetic correlations have been either phenomenological [11], or numerical [16, 18, 19].

In the present work we are able to calculate the mag- 
netic correlation function analytically for temperatures well above the characteristic dipolar energy, believed to be the dominant interaction in these systems [22]. These correlations are included in the full treatment of the Boltzmann kinetic equation. Because dipolar interactions are not isotropic, we predict a weak dependence of $\Delta \rho$ on the angle $\theta_{h}$ between the electrical and magnetic fields. This should not be confused with the anisotropic magnetoresistance (AMR) seen in systems with a ferromagnetic percolating cluster or arising from lattice distortions due to film substrate stresses [23, 24].

In Sec. II we discuss the physical parameters for the considered system and the limits for validity of the related model. In Sec. III A the spin-dependent Boltzmann equation for this model is formulated and in Sec. IIIB the explicit solution is obtained for the distribution function, emphasizing the importance of spin flip scattering in a situation where forward scattering dominates. The expressions for resistivity $\rho$ and magnetoresistance ratio $\Delta \rho / \rho$ with various observable dependencies (including a weak dependence of $\Delta \rho / \rho$ on the angle $\theta_{h}$ ) are presented in section IV. Finally, a general discussion and some comparisons to previous theories and experimental results are presented in section $\mathrm{D}$.

Partial preliminary results have been already reported in Refs. [25, 26], giving a general formulation of the model and the expression for GMR in a particular geometry (parallel electric and magnetic fields). Here we give a more detailed presentation of the solution of Boltzmann equation, generalize it to any angle between electric and magnetic fields and present a full discussion of the implications of our results.

\section{DEFINITION OF PHYSICAL SYSTEM AND MODEL}

We consider a metallic system of identical magnetic spherical granules of diameter $d$, randomly embedded with volume fraction $f$ into non-magnetic metallic matrix. This is a reasonable approximation to real granular alloys, like $\mathrm{Fe} C u$, $\mathrm{Co} A u$, etc., where the magnetic component by transition ( $\mathrm{T}$ ) metal is mostly aggregated in standard granules (having sizes only slightly dispersed around the mean value and shapes of polyhedra faceted along low-index crystalline planes, close to spheres). Conductance in such systems is mainly realized by $s$-like electrons, shared between noble metal $(\mathrm{N})$ matrix and granules, and they are scattered by magnetic $d$-electrons only present in the granules (it should be noted that both $\mathrm{T}$ - and N-atoms occupy the sites in a common crystalline lattice). At room temperature, $d$-electrons are distributed in split spin sub-bands and provide almost saturated, uniform magnetization within each granule. However this magnetization can be randomly oriented in different granules. Since Fermi $s$-electron has in general different velocities in $\mathrm{N}$ - and T-metals and its spin is subjected to some contact interaction with the polarized spins of $d$-electrons (whole bands), the scattering operator can be modeled by the form [17, 25, 26]:

$$
W_{\sigma \sigma^{\prime}}(\mathbf{r})=\sum_{j} \chi\left(\mathbf{r}-\mathbf{R}_{\mathbf{j}}\right)\left(U \delta_{\sigma \sigma^{\prime}}+I \boldsymbol{\tau}_{\sigma \sigma^{\prime}} \cdot \boldsymbol{\mu}_{j}\right) .
$$

Here $U$ and $I$ are the parameters of potential and spindependent scattering, $\boldsymbol{\tau}_{\sigma \sigma^{\prime}}$ is the $s$-electron spin operator. The relevant variable for $j$ th granule, the unit vector $\boldsymbol{\mu}_{j}$ along its magnetic moment, is considered classical and invariable at electron scattering events, since the net granule moment $\mu_{0}$ typically amounts to $\sim 10^{4} \mu_{\mathrm{B}}$ and its coupling to the environment should be stronger than an energy transfer at electron spin flip. Thus the model, Eq. 1, implies transitions between different spin channels due to spin precession in the field of classical magnetization within a granule, rather than due to less probable spin flips by individual atomic scatterers. The function $\chi\left(\mathbf{r}-\mathbf{R}_{j}\right)$ in the simplest approximation is 1 when $\left|\mathbf{r}-\mathbf{R}_{j}\right|<r_{0}$, ( $r_{0}$ being the radius of a granule) and zero otherwise. It is the Fourier transform of this function (see below) that effectively accounts for a distinguished role of interface scattering, while it was to be specially introduced into the incoherent scattering schemes. The scattering potential, Eq. 11, is also easily generalized to different sized granules. A similar model, which also considered coherent scattering by the granules, was formerly proposed by Kim et al [27]. However, these authors assumed a dipolar (instead of exchange) coupling between granule magnetization and charge carriers spin and did not include magnetic correlations between granules.

In what follows, some important relations will be used between the characteristic length scales for this problem: the Fermi wavelength $\lambda_{\mathrm{F}}=2 \pi / k_{\mathrm{F}}$, the mean granule diameter $d$, the mean intergranule distance $D=$ $(\pi / 6 f)^{1 / 3} d$, and the mean free path $\ell$ for conduction electrons. Namely, we consider them to obey the following sequence of inequalities

$$
\ell \gg D>d \gg \lambda_{\mathrm{F}}
$$

which is rather realistic for experimental systems (see for instance [10]).

A particular physical consequence of the relation $d \gg$ $\lambda_{\mathrm{F}}$ is that scattering is dominated by small angles as follows from standard diffraction arguments. We shall see that in this situation the spin flip scattering has increased importance in determining the structure of the stationary electronic distribution function.

\section{BOLTZMANN KINETIC EQUATION}

\section{A. Spin-dependent distribution functions}

We use the description of non-equilibrium electronic state of a granular system, related to the scattering potential, Eq. 1, and subjected to external electric and 
magnetic fields, in terms of spin-dependent distribution functions $f_{\mathbf{k} \sigma}$ obeying the kinetic Boltzmann equation (BE)

$$
\begin{gathered}
\frac{\partial f_{\mathbf{k} \sigma}}{\partial t}-\mathbf{v}_{\mathbf{k}} \\
\cdot \nabla_{\mathbf{r}} f_{\mathbf{k} \sigma}-\frac{e}{\hbar}\left(\mathbf{E}+\frac{1}{c} \mathbf{v}_{\mathbf{k}} \times \mathbf{H}\right) \cdot \nabla_{\mathbf{k}} f_{\mathbf{k} \sigma}+ \\
+\sum_{\mathbf{k}^{\prime}, \sigma^{\prime}}\left(f_{\mathbf{k}^{\prime} \sigma^{\prime}}-f_{\mathbf{k} \sigma}\right) W_{\mathbf{k} \sigma, \mathbf{k}^{\prime} \sigma^{\prime}}=0
\end{gathered}
$$

where $\mathbf{v}_{\mathbf{k}}$ is the conduction electron velocity and $W_{\mathbf{k} \sigma, \mathbf{k}^{\prime} \sigma^{\prime}}$ its transition probability from $\mathbf{k}^{\prime} \sigma^{\prime}$ to $\mathbf{k} \sigma$ state per unit time. In absence of fields, a trivial steady state solution holds $f_{\mathbf{k} \sigma} \equiv f_{\mathbf{k}}^{0}=\left\{\exp \left[\beta\left(\varepsilon_{\mathbf{k}}-\varepsilon_{\mathrm{F}}\right)\right]\right\}^{-1}$, describing the spin degenerate Fermi sphere. The electric field distorts the Fermi surface (FS) by shifting the Fermi sphere and the scattering redistributes electrons, a stationary distribution resulting from a balance between these two mechanisms. The current density by two spin channels is given by

$$
\mathbf{j}=-e \sum_{\mathbf{k}, \sigma} \mathbf{v}_{\mathbf{k}} f_{\mathbf{k} \sigma}
$$

and in absence of spin flip scattering the up and down spin FS are independent. In this case, the $\sigma$ spin FS distortion is proportional to $\tau_{\sigma}$, the corresponding relaxation time, and the conductivity is therefore proportional to $\tau_{\uparrow}+\tau_{\downarrow}$, a result used in 10, 12, 17, and in all calculations in the context of two-current model. Evidently, scattering between identical spin states is ineffective in relaxing the distortion of the FS if the angle of scattering is small. The characteristic transport factor $1-\cos \theta$ in our case can be estimated as

$$
1-\cos \theta \approx \frac{\theta^{2}}{2} \sim\left(\lambda_{\mathrm{F}} / d\right)^{2}
$$

since the diffraction angle $\theta \approx \lambda_{\mathrm{F}} / d \ll 1$.

However, if the spin flip scattering is present, it contributes very effectively, without the $1-\cos \theta$ factor, to the relaxation of the difference between the up and down spin FS distortions. So, when scattering in mostly in the forward direction, the spin flip scattering forces the up and down spin FS distortions to be almost identical. One then finds that the transport time is given by

$$
\frac{1}{\tau_{\operatorname{tr}}}=\frac{1}{2}\left(\frac{1}{\tau_{\uparrow}}+\frac{1}{\tau_{\downarrow}}\right)
$$

i.e. the rates, and not the times, must be averaged. We also include the effect of correlations between granule magnetic moments in a way that the scattering kernel of $\mathrm{BE}$ involves the connected correlation functions

$$
C_{\alpha}(\mathbf{q})=\left\langle\sum_{j(\neq i)} C_{i j}^{\alpha} \mathrm{e}^{-i \mathbf{q} \cdot \mathbf{R}_{j i}}\right\rangle_{\mathrm{R}}
$$

where $C_{i j}^{\alpha}=\left\langle\boldsymbol{\mu}_{i}^{\alpha} \cdot \boldsymbol{\mu}_{j}^{\alpha}\right\rangle-\left\langle\boldsymbol{\mu}_{i}^{\alpha}\right\rangle \cdot\left\langle\boldsymbol{\mu}_{j}^{\alpha}\right\rangle,\langle\cdots\rangle$ denotes the thermal average and $\langle\cdots\rangle_{\mathrm{R}}$ the average over granule positions. Correlations induced by the dipolar interaction are not isotropic and depend on the angle between $\mathbf{q}$ and the external magnetic field. As will be shown below, this results in a certain dependence of the magnetoresistance on the angle between current and magnetic field.

\section{B. Solution of kinetic equation}

In presence of fields, we define a usual expansion to linearize $\mathrm{BE}$

$$
f_{\mathbf{k} \sigma} \equiv f_{\mathbf{k}}^{0}-\frac{\partial f_{\mathbf{k}}^{0}}{\partial \varepsilon_{\mathbf{k}}} \phi_{\mathbf{k} \sigma} \approx f^{0}\left(\varepsilon_{\mathbf{k}}-\phi_{\mathbf{k} \sigma}\right),
$$

that is $\phi_{\mathbf{k} \sigma}$ can be interpreted as the FS deformation. Then Eq. 4 is expressed as

$$
\mathbf{j}=-2 e \sum_{\mathbf{k}} \mathbf{v}_{\mathbf{k}}\left(-\frac{\partial f_{\mathbf{k}}^{0}}{\partial \varepsilon_{\mathbf{k}}}\right) \phi_{\mathbf{k}} .
$$

where $\phi_{\mathbf{k}} \equiv \frac{1}{2} \sum_{\sigma} \phi_{\mathbf{k} \sigma}$. Now BE, Eq. 3, for spatially uniform steady state and with neglect of orbital effects by magnetic field, can be reduced to an integral equation for $\phi_{\mathbf{k} \sigma}$

$$
e \mathbf{v}_{\mathbf{k}} \cdot \mathbf{E}=\sum_{\sigma^{\prime}} \int d \Omega_{\mathbf{k}^{\prime}} \omega_{\sigma \sigma^{\prime}}\left(\mathbf{k}, \mathbf{k}^{\prime}\right) \phi_{\mathbf{k}^{\prime} \sigma^{\prime}}
$$

The angular integration in Eq. 7 is over FS and the kernel $\omega_{\sigma \sigma^{\prime}}\left(\mathbf{k}, \mathbf{k}^{\prime}\right)$ is

$$
\begin{aligned}
\omega_{\sigma \sigma^{\prime}}\left(\mathbf{k}, \mathbf{k}^{\prime}\right) & =\sum_{\sigma^{\prime \prime}} \int \frac{d \Omega_{\mathbf{k}^{\prime \prime}}}{4 \pi} \rho_{\mathrm{F}} \Gamma_{\sigma \sigma^{\prime \prime}}\left(\mathbf{k}, \mathbf{k}^{\prime \prime}\right) \delta\left(\Omega_{\mathbf{k}}-\Omega_{\mathbf{k}^{\prime}}\right) \delta_{\sigma \sigma^{\prime}} \\
& -\frac{1}{4 \pi} \rho_{\mathrm{F}} \Gamma_{\sigma \sigma^{\prime}}\left(\mathbf{k}, \mathbf{k}^{\prime}\right)
\end{aligned}
$$

where $\rho_{\mathrm{F}}$ is the Fermi density of states. The transition probability density $\Gamma_{\sigma \sigma^{\prime}}\left(\mathbf{k}, \mathbf{k}^{\prime}\right)$ can be written like in Refs. [17, 25, 26] using the Fermi's golden rule

$$
\frac{1}{V} \Gamma_{\sigma \sigma^{\prime}}\left(\mathbf{k}, \mathbf{k}^{\prime}\right)=\frac{2 \pi}{\hbar}\left|\left\langle\mathbf{k}, \sigma|\hat{W}| \mathbf{k}^{\prime}, \sigma^{\prime}\right\rangle\right|^{2}
$$

where $V$ is the sample volume.

For the common case of point-like scatterers, the validity of Born approximation only requires that the relevant energy scales for perturbation, $U$ and $I$, be small compared to the Fermi energy $\varepsilon_{\mathrm{F}}$ [28]. But in our case, the finite size of scatterers also needs some consideration. In a naïve view, the strength of perturbation could be represented by the scattering rates (see below)

$$
\gamma_{U}^{2}=\frac{2 \pi}{\hbar} V_{0} U^{2} \rho_{\mathrm{F}}, \quad \gamma_{I}^{2}=\frac{2 \pi}{\hbar} V_{0} I^{2} \rho_{\mathrm{F}},
$$

where $V_{0}=\pi d^{3} / 6$ is the granule volume. For a relevant choice of parameters: $V_{0} \sim 10 \mathrm{~nm}^{3}, U \sim I \sim 0.3 \mathrm{eV}$, $\rho_{\mathrm{F}} \sim 10 \mathrm{eV}^{-1} \mathrm{~nm}^{-3}, \varepsilon_{\mathrm{F}} \sim 5 \mathrm{eV}$, one has a large ratio $\hbar \gamma_{I}^{2} / \varepsilon_{\mathrm{F}}>1$. However, this estimate ignores the fact that the scattering is mostly in forward direction. Taking 
this into account, the lifetime of a momentum state is estimated as :

$$
\hbar / \tau \sim f\left(\gamma_{U}^{2}+\gamma_{I}^{2}\right) /\left(k_{\mathrm{F}} d\right)^{2} \approx f\left(U^{2}+I^{2}\right) k_{\mathrm{F}} d / \varepsilon_{\mathrm{F}} .
$$

Hence the condition of weak scattering $\hbar / \tau \ll \varepsilon_{\mathrm{F}}$ remains valid even for values of $U$ and $I$ as large as $\sim 1 \mathrm{eV}$.

Then BE, Eq. ॠ, can be rewritten as an operator equation in the space of functions defined on FS,

$$
\sum_{\sigma^{\prime}} \hat{\omega}_{\sigma \sigma^{\prime}}\left|\phi_{\sigma^{\prime}}\right\rangle=\left|\phi_{\mathrm{E}}\right\rangle
$$

where $\left|\phi_{\mathrm{E}}\right\rangle$ denotes the driving term and $\left|\phi_{\sigma}\right\rangle$ the FS distortion for spin $\sigma$.

We use the angular momentum basis $|\ell m\rangle$ and define the coefficients

$$
\phi_{\ell m \sigma} \equiv\left\langle\ell m \mid \phi_{\sigma}\right\rangle \equiv \phi_{\ell m}+\sigma \eta_{\ell m},
$$

so that

$$
\phi_{\mathbf{k} \sigma}=\sum_{\ell, m} \phi_{\ell m \sigma} \mathrm{Y}_{\ell}^{m}(\hat{\mathbf{k}}) .
$$

Note that since the driving term of $\mathrm{BE}$ is $e \mathbf{v}_{\mathbf{k}} \cdot \mathbf{E} \propto$ $e v_{\mathbf{k}} E \mathrm{Y}_{1}^{0}(\hat{\mathbf{k}})$, only the $\ell=1, m=0$ component of $\left|\phi_{\mathrm{E}}\right\rangle$ is non zero. As was already mentioned, correlations induced by dipolar interactions lead to a dependence of the scattering kernel on the angle between the momentum transfer vector $\mathbf{q} \equiv \mathbf{k}^{\prime}-\mathbf{k}$ and the external magnetic field, and it is convenient to separate out its (small) anisotropic part from the main isotropic one:

$$
\begin{aligned}
& \Gamma_{\sigma \sigma^{\prime}}\left(\mathbf{k}, \mathbf{k}^{\prime}\right)=\Gamma_{\sigma \sigma^{\prime}}^{(i)}(q)+\Gamma_{\sigma \sigma^{\prime}}^{(a)}(\mathbf{q}), \\
& \hat{\omega}_{\sigma \sigma^{\prime}}\left(\mathbf{k}, \mathbf{k}^{\prime}\right)=\hat{\omega}_{\sigma \sigma^{\prime}}^{(i)}(q)+\hat{\omega}_{\sigma \sigma^{\prime}}^{(a)}(\mathbf{q}) .
\end{aligned}
$$

Let us first restrict consideration to the isotropic scattering kernel, $\omega_{\sigma \sigma^{\prime}}^{(i)}(q)$, which depends only on the angle between $\mathbf{k}$ and $\mathbf{k}^{\prime}$. The resulting operator in Eq. 11 is diagonal in the spherical harmonic basis with $z$ axis along the only distinguished direction of electric field $\mathbf{E}$ (E-basis). Note that in this case the quantization axis for electron spin operator $\widehat{\tau}$ can be chosen arbitrary. Then, presenting the relevant solution as $\phi_{10 \sigma}^{(i)}=\phi_{10}^{(i)}+\sigma \eta_{10}^{(i)}$ we obtain its components $\phi_{10}^{(i)}$ and $\eta_{10}^{(i)}$ from

$$
\begin{gathered}
\omega_{s s}^{(i)} \phi_{10}^{(i)}+\omega_{s a}^{(i)} \eta_{10}^{(i)}=\sqrt{4 \pi / 3} e v_{\mathbf{k}} E, \\
\omega_{a s}^{(i)} \phi_{10}^{(i)}+\omega_{a a}^{(i)} \eta_{10}^{(i)}=0,
\end{gathered}
$$

all other components of the FS distortion being zero. We should emphasize that, in any case, only the $\ell=1 \mathrm{com}$ ponents of $\phi_{\mathbf{k} \sigma}$ contribute to the current. The rates appearing in Eq. 13 are given by

$$
\begin{aligned}
& \omega_{s s}^{(i)}=\frac{1}{2} \sum_{\sigma \sigma^{\prime}}\left\langle 10\left|\hat{\omega}_{\sigma \sigma^{\prime}}^{(i)}\right| 10\right\rangle, \\
& \omega_{s a}^{(i)}=\frac{1}{2} \sum_{\sigma \sigma^{\prime}} \sigma^{\prime}\left\langle 10\left|\hat{\omega}_{\sigma \sigma^{\prime}}^{(i)}\right| 10\right\rangle,
\end{aligned}
$$

$$
\begin{aligned}
\omega_{a s}^{(i)} & =\frac{1}{2} \sum_{\sigma \sigma^{\prime}} \sigma\left\langle 10\left|\hat{\omega}_{\sigma \sigma^{\prime}}^{(i)}\right| 10\right\rangle, \\
\omega_{a a}^{(i)} & =\frac{1}{2} \sum_{\sigma \sigma^{\prime}} \sigma \sigma^{\prime}\left\langle 10\left|\hat{\omega}_{\sigma \sigma^{\prime}}^{(i)}\right| 10\right\rangle,
\end{aligned}
$$

or, more explicitly,

$$
\begin{aligned}
& \omega_{s s}^{(i)}=\frac{\rho_{\mathrm{F}}}{2} \sum_{\sigma, \sigma^{\prime}} \int \frac{d \Omega_{\mathbf{k}^{\prime}}}{4 \pi} \Gamma_{\sigma \sigma^{\prime}}^{(i)}\left(\left|\mathbf{k}-\mathbf{k}^{\prime}\right|\right) \quad\left(1-\cos \left(\theta_{\mathbf{k k}^{\prime}}\right)\right), \\
& \omega_{s a}^{(i)}=\frac{\rho_{\mathrm{F}}}{2} \sum_{\sigma, \sigma^{\prime}} \int \frac{d \Omega_{\mathbf{k}^{\prime}}}{4 \pi} \Gamma_{\sigma \sigma^{\prime}}^{(i)}\left(\left|\mathbf{k}-\mathbf{k}^{\prime}\right|\right)\left(\sigma-\sigma^{\prime} \cos \left(\theta_{\mathbf{k k}^{\prime}}\right)\right), \\
& \omega_{a s}^{(i)}=\frac{\rho_{\mathrm{F}}}{2} \sum_{\sigma, \sigma^{\prime}} \int \frac{d \Omega_{\mathbf{k}^{\prime}}}{4 \pi} \Gamma_{\sigma \sigma^{\prime}}^{(i)}\left(\left|\mathbf{k}-\mathbf{k}^{\prime}\right|\right) \sigma\left(1-\cos \left(\theta_{\mathbf{k k}^{\prime}}\right)\right), \\
& \omega_{a a}^{(i)}=\frac{\rho_{\mathrm{F}}}{2} \sum_{\sigma, \sigma^{\prime}} \int \frac{d \Omega_{\mathbf{k}^{\prime}}}{4 \pi} \Gamma_{\sigma \sigma^{\prime}}^{(i)}\left(\left|\mathbf{k}-\mathbf{k}^{\prime}\right|\right)\left(1-\sigma \sigma^{\prime} \cos \left(\theta_{\mathbf{k k}^{\prime}}\right)\right) .
\end{aligned}
$$

Solving Eqs. 13, we obtain $\phi_{10}^{(i)}=\sqrt{4 \pi / 3} e v_{\mathbf{k}} E \tau_{t r}^{(i)}$ where

$$
\tau_{t r}^{(i)-1}=\omega_{s s}^{(i)}-\frac{\omega_{s a}^{(i)} \omega_{a s}^{(i)}}{\omega_{a a}^{(i)}}
$$

is the transport relaxation time in isotropic approximation. Introducing $\phi_{\mathbf{k}}$ into Eq. 6 in the same approximation:

$$
\phi_{\mathbf{k}}^{(i)}=\phi_{10}^{(i)} \mathrm{Y}_{1}^{0}(\hat{\mathbf{k}})=e \mathbf{v}_{\mathbf{k}} \cdot \mathbf{E} \tau_{t r}^{(i)},
$$

we arrive in a standard way at the Drude resistivity

$$
\rho=\frac{m_{e}}{n_{e} e^{2} \tau_{t r}^{(i)}} .
$$

As stated in Sec. II and explicitly shown below, the scattering probability $\Gamma_{\sigma \sigma^{\prime}}\left(\mathbf{k}, \mathbf{k}^{\prime}\right)$ is dominated by small angles $\theta_{\mathbf{k k}^{\prime}}$. As a result, the integrals in Eqs. 14 that involve the factor $1+\cos \theta$ are larger than those with $1-\cos \theta$ by a factor of $\left(k_{\mathrm{F}} d\right)^{2}$. They appear only in $\omega_{a a}$ (and cancel in $\omega_{s a}$ ) so that

$$
\tau_{t r}^{(i)-1} \approx \omega_{s s}^{(i)} .
$$

Considering the definitions of Eq. 14, we can easily conclude that

$$
\omega_{s s}^{(i)}=\frac{1}{2}\left(\tau_{\uparrow \uparrow}^{(i)-1}+\tau_{\downarrow \downarrow}^{(i)-1}+2 \tau_{\downarrow \uparrow}^{(i)-1}\right)
$$

where the rates $\tau_{\sigma \sigma^{\prime}}^{(i)}-1$ are defined as

$$
\tau_{\sigma \sigma^{\prime}}^{(i)}-1=\rho_{\mathrm{F}} \int \frac{d \Omega_{\mathbf{k}^{\prime}}}{4 \pi} \Gamma_{\sigma \sigma^{\prime}}^{(i)}\left(\left|\mathbf{k}-\mathbf{k}^{\prime}\right|\right)\left(1-\cos \left(\theta_{\mathbf{k k}^{\prime}}\right)\right) .
$$

Eq. 17 expresses the fact, already discussed in Sec. III A, that up and down spin FS have almost the same deformation and so the relaxation rate of the mean of the two deformations is just the mean of corresponding rates. For 
a typical value $k_{\mathrm{F}} d \approx 40$ the neglected term in Eq. 15 turns out to be about $0.5 \%$ of the term retained.

The weak anisotropic term of the scattering kernel, $\hat{\omega}_{\sigma \sigma^{\prime}}^{(a)}$, due to the correlation between magnetic moments, can be easily included into the present treatment in a perturbative way. Then Eq. 11 reads

$$
\sum_{\sigma^{\prime}}\left(\hat{\omega}_{\sigma \sigma^{\prime}}^{(i)}\left|\phi_{\sigma^{\prime}}\right\rangle+\hat{\omega}_{\sigma \sigma^{\prime}}^{(a)}\left|\phi_{\sigma^{\prime}}\right\rangle\right)=\left|\phi_{\mathrm{E}}\right\rangle,
$$

and the solution can be written as $\left|\phi_{\sigma}\right\rangle=\left|\phi_{\sigma}^{(i)}\right\rangle+\left|\phi_{\sigma}^{(a)}\right\rangle$, where $\left|\phi_{\sigma}^{(a)}\right\rangle$ is a small anisotropic perturbation. To lowest non zero order in it, we have

$$
\sum_{\sigma^{\prime}}\left(\hat{\omega}_{\sigma \sigma^{\prime}}^{(i)}\left|\phi_{\sigma}^{(a)}\right\rangle+\hat{\omega}_{\sigma \sigma^{\prime}}^{(a)}\left|\phi_{\sigma^{\prime}}^{(i)}\right\rangle\right)=0 .
$$

Projecting out the $\ell=1$ and $m=0$ components and using the facts that $\hat{\omega}_{\sigma \sigma^{\prime}}^{(i)}$ is diagonal and the only non zero component in the unperturbed solution $\left|\phi_{\sigma}^{(i)}\right\rangle$ is that with $\ell=1, m=0$, we can solve this equations for the coefficients $\phi_{10 \sigma}^{(a)}=\left\langle 10 \mid \phi_{\sigma}^{(a)}\right\rangle=\phi_{10}^{(a)}+\sigma \eta_{10}^{(a)}$. A simplification similar to that leading to Eq. 16 also applies here and we obtain

$$
\phi_{10}^{(a)}=-\frac{\omega_{s s}^{(a)}}{\omega_{s s}^{(i)}} \phi_{10}^{(i)}
$$

where $\omega_{s s}^{(a)}$ is defined in a similar way to $\omega_{s s}^{(i)}$ in Eq. 14 . Hence the transport time $\tau_{t r}$ is changed by a factor $1-$ $\omega_{s s}^{(a)} / \omega_{s s}^{(i)}$ compared to $\tau_{t r}^{(i)}$, Eq. 16, and to the order of accuracy that we are working in Eq. 18, we may write

$$
\tau_{t r}^{-1}=\omega_{s s}^{(i)}+\omega_{s s}^{(a)} .
$$

One might question at this point, whether it is legitimate to include this last correction while neglecting the second term in Eq. 15. It should be stressed however that the two terms in Eq. 19 are of the same order with respect to the small parameter $1 /\left(k_{\mathrm{F}} d\right)$. So our theory is consistently a lowest non zero order theory in this small parameter. On the more practical side, we will see that for typical parameter values this correction, arising from spin correlations, can in fact be more important than the terms neglected in Eq. 15 .

\section{CALCULATION OF TRANSPORT RATES}

\section{A. Isotropic Kernel}

The principles for the calculation of the scattering rates have already been spelled out in Ref. [17. The explicit squaring matrix element in Eq. 9 is

$$
\left|\left\langle\mathbf{k}, \sigma|\hat{W}| \mathbf{k}^{\prime}, \sigma^{\prime}\right\rangle\right|^{2}=\frac{V_{0}^{2}}{V^{2}} \psi^{2}\left(\frac{q d}{2}\right)\left|\sum_{j} e^{-i \mathbf{q} \cdot \mathbf{R}_{j}} W_{\sigma \sigma^{\prime}}^{j}\right|^{2}
$$

where the function $\psi(x)=3(\sin (x)-x \cos (x)) / x^{3}$ is the structure factor of a sphere, the Fourier image of the function $\chi(r)$, and $W_{\sigma \sigma^{\prime}}^{j}=U \delta_{\sigma \sigma^{\prime}}+I \boldsymbol{\tau}_{\sigma \sigma^{\prime}} \cdot \boldsymbol{\mu}_{j}$. It is due to the presence of factors $\psi^{2}(q d / 2)$ in the integrals of Eq. 14 that only values of $q \leq d^{-1}$ contribute significantly to the scattering. Evidently, Eq. 20 should be averaged with respect to the random positions $\mathbf{R}_{i}$ of the granules and over the thermal distribution of their moments $\boldsymbol{\mu}_{i}$. The latter can be done in the basis with $z$ axis along the external magnetic field $\mathbf{H}$ (H-basis), where only the $z$ component of a magnetic moment has non zero average $\left\langle\mu_{i}^{z}\right\rangle \equiv\left\langle\mu_{z}\right\rangle$.

We separate out contribution involving a single granule, and write averages of products of magnetic moments of diferent granules as products of averages plus connected correlation functions, Eq. 5. It is then straightforward to obtain the explicit formulas for diagonal and nondiagonal components of isotropic and anisotropic parts of the scattering rates, Eq. 12 :

$$
\begin{aligned}
\rho_{\mathrm{F}} \Gamma_{\sigma \sigma}^{(i)}(q) & =f \psi^{2}\left(\frac{q d}{2}\right)\left(\gamma_{U}^{2}(1+g(q))+\right. \\
& +2 \sigma \gamma_{U} \gamma_{I}\left\langle\mu_{z}\right\rangle(1+g(q)) \\
& \left.+\gamma_{I}^{2}\left(\left\langle\mu_{z}^{2}\right\rangle+g(q)\left\langle\mu_{z}\right\rangle^{2}\right)\right), \\
\rho_{\mathrm{F}} \Gamma_{\sigma,-\sigma}^{(i)}(q) & =f \psi^{2}\left(\frac{q d}{2}\right) \gamma_{I}^{2}\left[1-\left\langle\mu_{z}^{2}\right\rangle\right],
\end{aligned}
$$

and

$$
\begin{gathered}
\rho_{\mathrm{F}} \Gamma_{\sigma \sigma}^{(a)}(\mathbf{q})=f \psi^{2}\left(\frac{q d}{2}\right) \gamma_{I}^{2} C_{\|}(\mathbf{q}) \\
\rho_{\mathrm{F}} \Gamma_{\sigma,-\sigma}^{(a)}(\mathbf{q})=f \psi^{2}\left(\frac{q d}{2}\right) \gamma_{I}^{2} C_{\perp}(\mathbf{q})
\end{gathered}
$$

Here $g(q)$ is the pair correlation function, already calculated in Refs. [17, 25, 26, using the excluded volume approximation

$$
\begin{aligned}
g(q) & \equiv \frac{1}{N}\left\langle\sum_{i \neq j} e^{-i \mathbf{q} \cdot\left(\mathbf{R}_{j}-\mathbf{R}_{i}\right)}\right\rangle_{\{R\}} \approx \\
& \approx \frac{f}{V_{0}} \int_{r>d} d \mathbf{r} \mathrm{e}^{-i \mathbf{q} \cdot \mathbf{r}}=-8 f \psi(q d)
\end{aligned}
$$

(a delta function at $q=0$ is neglected because it gives a zero contribution to the integrals over $q$ ). The correlation functions $C_{\|}(\mathbf{q}) \equiv C_{z}(\mathbf{q})$ and $C_{\perp}(\mathbf{q}) \equiv C_{x}(\mathbf{q})+C_{y}(\mathbf{q})$ and the relevant average $\left\langle\mu_{z}\right\rangle$ are calculated in the same approximation in Appendix B.

The angular integrations appearing in Eq. 14 can now be transformed to integrals over the momentum transfer $q$. They can be classified in terms of inverse powers of the large parameter $k_{\mathrm{F}} d$ (see Appendix A). The terms, involving the factor $1+\cos \theta$, are of the order $\left(\gamma_{U}^{2}, \gamma_{I}^{2}\right) f /\left(k_{\mathrm{F}} d\right)^{2}$ whereas the factors involving the factor $1-\cos \theta \propto q^{2}$ are smaller by a factor $1 /\left(k_{\mathrm{F}} d\right)^{2}$ i.e., of the order $\left(\gamma_{U}^{2}, \gamma_{I}^{2}\right) f /\left(k_{\mathrm{F}} d\right)^{4}$.

In the absence of correlations the result for the transport rate is quite simple (see Eq. 15) 


$$
\tau_{t r}^{-1}=\frac{4 f}{\left(k_{\mathrm{F}} d\right)^{4}}\left(\alpha \gamma^{2}-\beta f \gamma_{I}^{2}\left\langle\mu_{z}\right\rangle^{2}\right)
$$

where $\gamma^{2}=\gamma_{U}^{2}+\gamma_{I}^{2}$ and the constants $\alpha=(9 / 2) \ln \left(k_{\mathrm{F}} d\right)$, and $\beta \approx 4.172$ are defined in Appendix A. Other (nonmagnetic) scattering mechanisms will give rise to an additive background contribution to the resistivity $\rho_{b}$ so that

$$
\rho=\rho_{b}+\frac{m_{e}}{n_{e} e^{2}} \frac{4 f}{\left(k_{\mathrm{F}} d\right)^{4}}\left(\alpha \gamma^{2}-\beta f \gamma_{I}^{2}\left\langle\mu_{z}\right\rangle^{2}\right)
$$

This result is identical to the first two terms of Eq. (18) in Ref. 177 and it gives a magnetoresistance proportional to the square of magnetization

$$
-\Delta \rho=\frac{m_{e}}{n_{e} e^{2}} \frac{4 \beta f^{2} \gamma_{I}^{2}}{\left(k_{\mathrm{F}} d\right)^{4}}\left\langle\mu_{z}\right\rangle^{2}
$$

Such proportionality of the magnetoresistance to $\left\langle\mu_{z}\right\rangle^{2}$, at this level of approximation is also obtained in all calculations in the two-current model 10,12 . Differences between these approaches will be discussed in more detail in Sec. V.

\section{B. Contribution of Correlations}

A calculation of the effect of correlations on transport requires explicit expressions for the correlation functions. In this article we consider only the high temperature limit of dipolar interactions, $k_{\mathrm{B}} T>\mu_{0}^{2} / D^{3}$ when the high temperature expansion is meaningful. The calculation gives (see Appendix B)

$$
C(\mathbf{q})=\frac{8 \pi f \mu_{0}^{2}}{3 V_{0} k_{\mathrm{B}} T} \mathcal{L}_{2}\left(\frac{\mu_{0} h}{k_{\mathrm{B}} T}\right) \psi(q d) P_{2}\left(\cos \theta_{\mathbf{q}, \mathbf{h}}\right)
$$

where $\mathcal{L}_{2}(s)=[\mathcal{L}(s) / s]^{2}-\left[\mathcal{L}^{\prime}(s)\right]^{2}$ and $\mathcal{L}(s)=\operatorname{coth}(s)-$ $1 / s$ is the Langevin function. The $\mathcal{L}_{2}$ factor is related to the common field effect on the magnetization. A more subtle field effect on the transport follows from the factor $P_{2}(x) \equiv\left(3 x^{2}-1\right) / 2$, depending on the angle $\theta_{\mathbf{q}, \mathbf{h}}$ between the scattering vector and the external magnetic field. This dependence results from the calculation of the correlator $C(\mathbf{q})$ using the H-basis (that where $z$-axis is along $\mathbf{H}$ ). It eventually introduces a dependence of the transport time on the angle $\theta_{h}$ between the current and the magnetic field (between E- and H-bases). Returning again to the E-basis (with $\mathbf{H}$ lying in the $x z$ plane) for integration in Eq. 8 and using the results of Eq. 21, one readily arrives at the following expression for the relevant rate $\omega_{s s}^{(a)}$ :

$$
\begin{aligned}
& \omega_{s s}^{(a)}=\frac{2}{3} \frac{f^{2} \gamma_{I}^{2} \mu_{0}^{2}}{V_{0} k_{\mathrm{B}} T} \mathcal{L}_{2}\left(\frac{\mu_{0} h}{k_{\mathrm{B}} T}\right) \times \\
& \times \int d \Omega_{\mathbf{k}} \int d \Omega_{\mathbf{k}^{\prime}} \psi^{2}\left(\frac{q d}{2}\right) \psi(q d) P_{2}\left(\cos \theta_{\mathbf{q}, \mathbf{h}}\right) \times \\
& \times\left[\left|Y_{1}^{0}\left(\theta_{\mathbf{k}}\right)\right|^{2}-Y_{1}^{0}\left(\theta_{\mathbf{k}^{\prime}}\right) Y_{1}^{0}\left(\theta_{\mathbf{k}}\right)\right] .
\end{aligned}
$$

The arguments of $\psi$ functions contain $q=2 k_{\mathrm{F}} \sin \left(\theta_{\mathbf{k k}^{\prime}} / 2\right)$ were $\theta_{\mathbf{k k}^{\prime}}$ is the angle between $\mathbf{k}$ and $\mathbf{k}^{\prime}$. The integrals over $\Omega_{\mathbf{k}}$ and $\Omega_{\mathbf{k}^{\prime}}$ in Eq. 26 are calculated using the addition theorem for spherical harmonics to give

$$
\omega_{s s}^{(a)}=-\frac{14 \pi}{15} \frac{\beta f^{2} \gamma_{I}^{2} \mu_{0}^{2}}{V_{0} k_{\mathrm{B}} T\left(k_{\mathrm{F}} d\right)^{4}} \mathcal{L}_{2}\left(\frac{\mu_{0} h}{k_{\mathrm{B}} T}\right) P_{2}\left(\cos \theta_{h}\right) .
$$

So, finally we obtain for the transport rate

$$
\begin{aligned}
\rho= & \rho_{b}+\frac{m_{e}}{n_{e} e^{2}} \frac{4 f}{\left(k_{\mathrm{F}} d\right)^{4}}\left\{\alpha \gamma^{2}-\right. \\
& \left.-\beta f \gamma_{I}^{2}\left[\left\langle\mu_{z}\right\rangle^{2}+\frac{7 \pi \mu_{0}^{2}}{30 V_{0} k_{\mathrm{B}} T} \mathcal{L}_{2}\left(\frac{\mu_{0} h}{k_{\mathrm{B}} T}\right) P_{2}\left(\cos \theta_{h}\right)\right]\right\}
\end{aligned}
$$

where again a background contribution, $\rho_{b}$, from non-magnetic scattering mechanisms (other impurities, phonons) is included. The magnetoresistance is then modified from Eq. 24 to

$$
-\Delta \rho=\frac{m_{e}}{n_{e} e^{2}} \frac{4 \beta f^{2} \gamma_{I}^{2}}{\left(k_{\mathrm{F}} d\right)^{4}}\left(\left\langle\mu_{z}\right\rangle^{2}+\frac{7 \pi}{30} \frac{T_{0}}{T} \mathcal{L}_{2}\left(\frac{\mu_{0} h}{k_{\mathrm{B}} T}\right) P_{2}\left(\cos \theta_{h}\right)\right)
$$

where $T_{0}$, the characteristic temperature of dipolar interactions, is

$$
T_{0}=\frac{\mu_{0}^{2}}{V_{0} k_{\mathrm{B}}}
$$

The last term in Eq. 28 describes the deviation from $\sim\left\langle\mu_{z}\right\rangle^{2}$ behavior of GMR due to dipolar magnetic correlations.

\section{DISCUSSION AND CONCLUSIONS}

When the coherence between individual scattering events inside a granule is disregarded [1, 10, 12] the relaxation rate of spin $\sigma$ electrons $\Delta_{\sigma} \equiv \tau_{\sigma}^{-1}=\Delta_{0}+\sigma \Delta_{1}$ is proportional to the volume fraction of granules, $f$, and $\Delta_{1}$ is proportional to the mean normalized magnetic moment $\left\langle\mu_{z}\right\rangle$. Furthermore, if magnetic scattering at the granule surface dominates, $\Delta_{1}$ is proportional to the surface to volume ratio of the granules i.e. inversely proportional to $d$, the diameter. In the two-current model the spin up and down electron subsystems carry current independently and the conductivity is given by

$$
\sigma=\frac{n_{e} e^{2}}{m_{e}}\left(\tau_{\uparrow}+\tau_{\downarrow}\right)
$$

or

$$
\rho(h)=\frac{m_{e}}{n_{e} e^{2}} \frac{\Delta_{0}^{2}-\Delta_{1}^{2}}{\Delta_{0}} .
$$

As a result the magnetoresistance is

$$
-\Delta \rho=\frac{m_{e}}{n_{e} e^{2}} \frac{\Delta_{1}^{2}}{\Delta_{0}} .
$$


From this a proportionality follows of $\Delta \rho$ to $\left\langle\mu_{z}\right\rangle^{2}, 1 / d$ and $f$, provided the resistance in zero field is also dominated by surface scattering at the granules $\left(\Delta_{0}\right.$ proportional to $f$ and $1 / d$ ).

In our model the entire granule scatters coherently. We believe this is reasonable, in view of the fact that the electronic mean free path can be larger than the granule diameter or even the inter-granular distance 110]. This corresponds to the homogeneous limit of Camblong et al [9]. Given the large size of the granules, compared to the Fermi wavelength, scattering is predominant in the forward direction, concentrated in a cone of angular size of order $\left(\lambda_{\mathrm{F}} / d\right)^{2} \sim 1 /\left(k_{\mathrm{F}} d\right)^{2}$. As we emphasized, spin flip scattering then enforces that the up and down spin FS distortions stay in step, and the resistivity is given by

$$
\rho=\frac{m}{n_{e} e^{2}}\left(\Delta_{\uparrow}+\Delta_{\downarrow}\right)
$$

The interference between scatterings in different granules gives a contribution to $\Delta_{\sigma}$ proportional to $\left\langle\boldsymbol{\mu}_{1} \cdot \boldsymbol{\mu}_{2}\right\rangle$ which, in the absence of correlations yields a $\left\langle\mu_{z}\right\rangle^{2}$ term proportional to $f^{2}$. Since in our calculation surface scattering is not specifically distinguished, one might expect the scattering cross section to be proportional to the volume of the granule $\sim d^{3}$. However, as we mentioned above, it is decreased by a factor of $1 /\left(k_{\mathrm{F}} d\right)^{2}$ by a standard diffraction argument. The contribution to the resistivity carries an extra factor $1 /\left(k_{\mathrm{F}} d\right)^{2}$ arising from the transport factor $1-\cos \theta$. As a result the resistivity is proportional to $1 / d$.

Given that our model corresponds to the homogeneous limit one could question whether it is capable of predicting the actual values of magnetoresistance ratio observed in experiments.

By defining a resistivity scale

$$
\Delta \rho_{m}=\frac{m_{e}}{n_{e} e^{2}} \frac{4 \beta f^{2} \gamma_{I}^{2}}{\left(k_{\mathrm{F}} d\right)^{4}}
$$

we may rewrite Eq. 28 in the form

$$
-\Delta \rho=\Delta \rho_{m}\left(\left\langle\mu_{z}\right\rangle^{2}+\frac{7 \pi T_{0}}{30 T} \mathcal{L}_{2}\left(\frac{\mu_{0} h}{k_{\mathrm{B}} T}\right) P_{2}\left(\cos \theta_{h}\right)\right) .
$$

The magnetic field dependent factor (within brackets) is zero for zero field and unity for saturating field, where correlations no longer contribute, so $\Delta \rho_{m}$ is the maximum value of $|\Delta \rho|$. The zero field resistance can be written as (see eq. 27)

$$
\rho(0)=\rho_{b}+\frac{\alpha}{\beta f}\left(1+\frac{\gamma_{U}^{2}}{\gamma_{I}^{2}}\right) \Delta \rho_{m}
$$

This gives an upper bound for the magnetoresistance ratio

$$
\frac{\Delta \rho_{m}}{\rho(0)} \leq \frac{\beta f}{\alpha}
$$

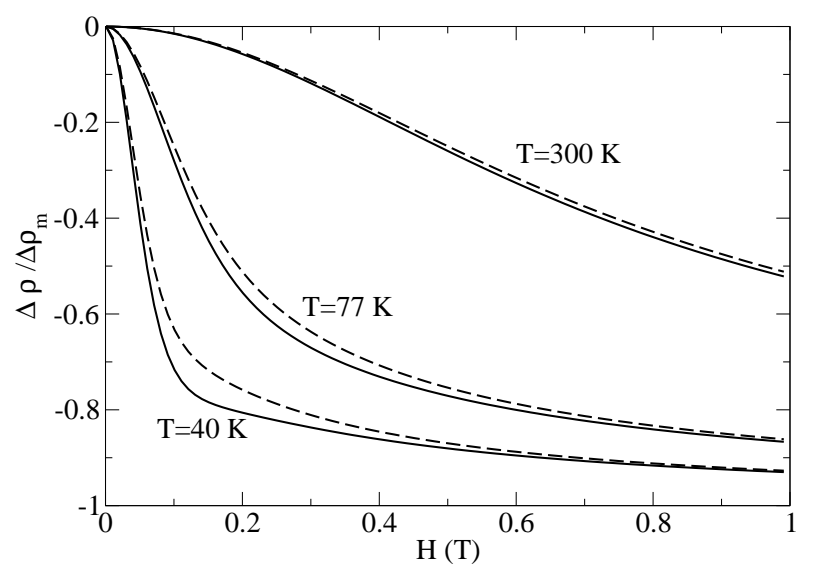

FIG. 1: Field dependent factor in the magnetoresistance as a function of magnetic field at various temperatures. Dashed lines - without dipolar correlation correction; full lines - with inclusion of dipolar correlation correction at parallel electric and magnetic fields $\left(T_{0}=107 \mathrm{~K}, \mu_{0}=1492 \mu_{\mathrm{B}}\right.$ and $f=0.2$ [10]).

The ratio $\beta / \alpha \approx 0.94 / \ln k_{\mathrm{F}} d$ varies between $0.31 \sim 0.23$ for $k_{\mathrm{F}} d$ in the range of $20 \sim 60$. This would imply $|\Delta \rho| / \rho \leq 0.3 f$, that is considerably smaller than what is usually observed below room temperature. The relatively small value of $\beta / \alpha$ is, however, a consequence of assuming a sharp granule interface in the function $\chi\left(\mathbf{r}-\mathbf{R}_{j}\right)$, which leads to a logarithmic factor in $\alpha$. To check this, we have also calculated with smoother density profiles and, for values of the interface thickness as small as $10 \%$ of the diameter [29], a rather realistic $\beta / \alpha \sim 0.8$ is obtained. For comparison, Wang and Xiao found maximum magnetoresistance ratios of the order of $5 \%$ at $300 \mathrm{~K}$ and $15 \%$ at $77 \mathrm{~K}$ in a sample of $\mathrm{Fe}_{20} \quad \mathrm{Ag}_{80}$ with $d=29$ $\AA$ [10]. These authors also found $\rho(0)$ to be inversely proportional to $d$, which means that the second term in Eq. 31 should dominate over $\rho_{b}$.

The effect of magnetic correlations on transport is contained in the second term of Eq. 30. Our treatment applies to dipolar interactions in the high temperature limit $\mu_{0}^{2} / D^{3} \ll k_{\mathrm{B}} T$, where $D$ is a typical minimum distance between granules. Since $D^{3}=V_{0} / f$, this condition is equivalent to $T \gg f T_{0}$. Using, as an example, the values of the sample mentioned above we estimate $T_{0} \approx 107 \mathrm{~K}$ and $f T_{0} \approx 21 \mathrm{~K}$. This shows that the high temperature approximation used to calculate the correlator, Eq. 25, is actually valid down to reasonably low temperatures.

To illustrate the effect of the correlation term, we compare in Fig. 11 the factor $\left\langle\mu_{z}\right\rangle^{2}+\left(7 \pi T_{0} / 30 T\right) \mathcal{L}_{2}\left(\mu_{0} h / k_{\mathrm{B}} T\right)$ for $\theta_{\mathbf{h}}=0$ (strongest correlation effect) with $\left\langle\mu_{z}\right\rangle^{2}$ alone, as functions of magnetic field for different temperatures (we used the parameters of the sample mentioned above, $T_{0}=107 \mathrm{~K}$ and $\left.\mu_{0}=1492 \mu_{\mathrm{B}}\right)$. As expected, the correlations give a larger correction (as much as 10\%) at lower 


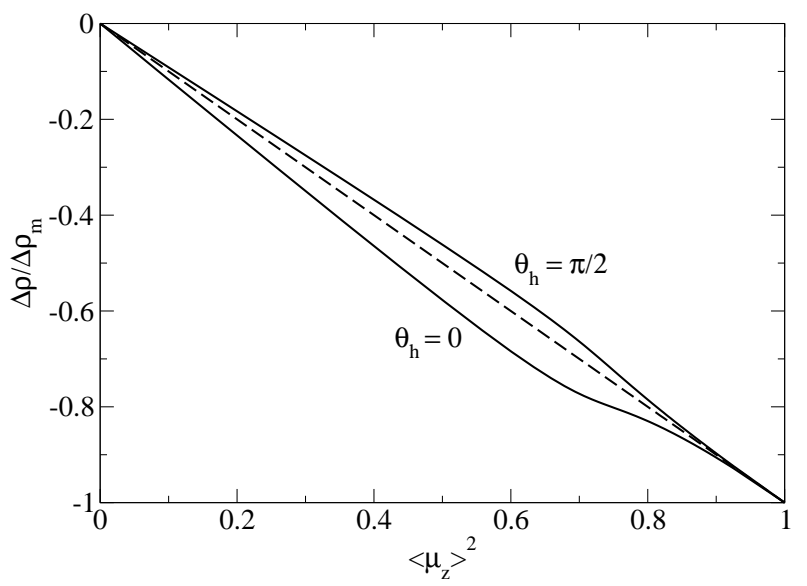

FIG. 2: Field dependent factor of magnetoresistance ratio, as a function of $\left\langle\mu_{z}\right\rangle^{2}$, for $T=40 \mathrm{~K}$ and the same sample parameters as in Fig. 1. The effect of dipolar correlations is seen in the curvature, which has opposite signs for parallel $\left(\theta_{h}=0\right)$ and perpendicular $\left(\theta_{h}=\pi / 2\right)$ electric and magnetic fields. Notice that for the angle $\theta_{h}=\arccos 1 / \sqrt{3} \approx 55^{\circ}$ the dipolar correlation correction turns zero and the straight line is recovered.

temperatures. A plot of magnetoresistance ratio $\Delta \rho / \rho$ vs $\left\langle\mu_{z}\right\rangle^{2}$ should be a straight line if the correlations are neglected. In Fig. 2 we illustrate this effect by representing $\Delta \rho / \Delta \rho_{m}$ vs $\left\langle\mu_{z}\right\rangle^{2}$ (see Eq. 30) for parallel $\left(\theta_{h}=0\right.$ ) and perpendicular $\left(\theta_{h}=\pi / 2\right)$ electric and magnetic fields. Note that the deviation from linear behaviour depends on temperature, granule parameters $\mu_{0}$ and $d$, through the temperature scale $T_{0}$ (Eq. 29), but not on the concentration $f$. Our result is remarkably similar to the one found by Kechrakos and Trohidou in a numerical calculation for mono-dispersive samples (see fig. 14 of their paper |19|). This effect, although small, has the interesting feature of depending on the angle between the electric and magnetic fields (see Fig.2) and having opposite signs in the case of parallel or perpendicular fields. The dependence on the relative orientation of electric and magnetic fields is due to the anisotropic nature of the dipolar interactions. The correlation between two granules is ferromagnetic for the moment components along the direction joining the granules but antiferromagnetic for the perpendicular components. The correlation correction vanishes and linearity of $\Delta \rho / \rho$ vs $\left\langle\mu_{z}\right\rangle^{2}$ is restored for the particular angle $\theta_{h}=\arccos 1 / \sqrt{3} \approx 55^{\circ}$. These features, together with a specific temperature dependence, should facilitate experimental detection of the dipolar correlation effect in GMR of granular metallic systems. It should be mentioned that granule size dispersion also leads to a nonlinear $\Delta \rho$ vs $\left\langle\mu_{z}\right\rangle^{2}$ [12, 17, 19 but always convex and $\theta_{h}$-independent. Both these kinds of non-linearity can coexist in real granular systems. At last we note that the correlation term by Eq. 30 is zero at zero magnetic field. However, at temperatures lower than $f T_{0}$, dipolar correlations cannot be treated within high temperature expansion, and it may happen then, that the zero field correlation effect remains finite. This in fact could rise the maximum magnetoresistance above the limit of Eq. 32, $\Delta \rho_{m}$. The anisotropy we predict may already have been noticed in $\mathrm{CuAg}$ films by Stearns and Cheng [30]. It should not be confused with usual AMR, due to skew magnetic scattering, observed in more concentrated systems [23].

To conclude, we would like to stress once more that these results follow from a peculiar structure of the electron distribution function, related to the fact that magnetic scattering is mostly in the forward direction. The relaxation rate of the difference between the up and down spin FS is the fastest one, leading to identical up and down spin FS distortions. The resulting model differs in some ways from the two-current model of magnetotransport, but seems to account equally well for the basic features of experiments. Some of the ideas presented here may also apply in other systems whenever large structures, but still smaller than electronic mean free path, dominate magnetic scattering.

\section{ACKNOWLEDGMENTS}

The authors would like to thank A.V. Vedyaev, B.L. Altshuler, A. H. Castro Neto, V.L. Belinicher, E. S. Lage and S.N. Dorogovtsev for very illuminating discussions. This work is supported by Fundação da Ciência e Tecnologia of Portugal, through project PRAXIS XXI 2/2.1/FIS/302/94. One of the authors (J. Viana Lopes) is supported by FCT grant $S F R H / B D / 1261 / 2000$.

\section{APPENDIX A}

The angular integrations, over the direction of $\mathbf{k}^{\prime}$, in Eqs. 14 can be transformed into integrations over the momentum transfer $q=2 k_{\mathrm{F}} \sin \theta_{\mathbf{k k}^{\prime}} / 2$. The term $\cos \theta_{\mathbf{k k}^{\prime}}=1-2\left(q / 2 k_{\mathrm{F}}\right)^{2}$ and the solid angle integration element, $d \Omega_{\mathbf{k}^{\prime}}=8 \pi q d q /\left(2 k_{\mathrm{F}}\right)^{2}$. Using of Eqs. 21 in Eqs. 14 involves the following integrals:

$$
\begin{gathered}
I(Q)=\int_{0}^{Q} x^{3} \psi^{2}(x) d x= \\
=\frac{9}{2}\left[\ln 2 Q+\gamma_{\mathrm{E}}-1-\operatorname{Ci}(2 Q)+\right. \\
\left.\quad+\frac{\sin 2 Q}{Q}-\frac{1-\cos 2 Q}{2 Q^{2}}\right], \\
J(Q)=\int_{0}^{Q} x^{3} \psi^{2}(x) \psi(2 x) d x= \\
=\frac{27}{20}[2 \ln 2-1+2 \operatorname{Ci}(2 Q)-2 \operatorname{Ci}(4 Q)- \\
-\frac{\sin 2 Q(1-\cos 2 Q)}{Q}\left(1+\frac{3}{4 Q^{2}}+\frac{1}{4 Q^{4}}\right)+
\end{gathered}
$$




$$
\left.+\frac{5+4 \cos 2 Q-\cos 4 Q}{8 Q^{2}}+\frac{\cos 2 Q-\cos 4 Q}{2 Q^{4}}\right] \text {, }
$$

where $\gamma_{\mathrm{E}} \approx 0.5772$ is Euler's gamma. The transition rates, Eq. 19, are simply expressed through these integrals:

$$
\begin{aligned}
\tau_{\operatorname{tr}}^{-1}= & \frac{4 f}{\left(k_{\mathrm{F}} d\right)^{4}}\left(\gamma^{2} I\left(k_{\mathrm{F}} d\right)-J\left(k_{\mathrm{F}} d\right) 8 f \gamma_{\mathrm{I}}^{2} \times\right. \\
& \left.\times\left(\left\langle\mu_{z}\right\rangle^{2}+\frac{7 \pi}{30} \frac{T}{T 0} \mathcal{L}_{2}\left(\frac{\mu_{0} h}{k_{\mathrm{B}} T}\right) P_{2}\left(\cos \theta_{\mathrm{h}}\right)\right)\right)
\end{aligned}
$$

and in the limit of $k_{\mathrm{F}} d \gg 1$ (taking into account the asymptotics of integral cosine $\mathrm{Ci}(x) \rightarrow \sin x / x)$ we have the result of Eq. 23 with $\alpha=(9 / 2) \ln \left(k_{\mathrm{F}} d\right)$ and $\beta=$ $(54 / 5)(2 \ln 2-1) \approx 4.172$.

\section{APPENDIX B}

The Hamiltonian for magnetic interactions of the granules is

$$
H=-\mu_{0} h \sum_{i} \mu_{i}^{z}+\mu_{0}^{2} \sum_{<i j>\alpha \beta} U_{i j}^{\alpha \beta} \mu_{i}^{\alpha} \mu_{j}^{\beta}
$$

where $h$ is the external magnetic field applied to the system (with $z$ axis rotated compared to the geometry in Sec. IVB) and $U_{i j}^{\alpha \beta}=\left(\delta_{\alpha \beta}-3 u_{i j}^{\alpha} u_{i j}^{\beta}\right) / r_{i j}^{3}$ (with the unit vector $\mathbf{u}_{i j}=\mathbf{r}_{i j} / r_{i j}$ ) is the dipolar interaction tensor. We made a standard high temperature expansion, with respect to the dipolar interaction energy, valid for temperatures for which the dipolar interaction is a small perturbation to the non-interacting Hamiltonean. $\left\langle\mu_{z}\right\rangle$

To the first order, we obtain for the magnetic moment

$$
\left\langle\mu_{i}^{z}\right\rangle=\mathcal{L}(s)\left(1-\frac{\mu_{0}^{2}}{k_{\mathrm{B}} T} \sum_{j \neq i} U_{i j}^{z z} \mathcal{L}^{\prime}(s)\right),
$$

[1] A.E. Berkowitz, J.R. Mitchell, M.J. Carey, A.P. Young, S. Zhang, F.E. Spada, F.T. Parker, A. Hutten, and G. Thomas, Phys. Rev. Lett. 68, 3745 (1992).

[2] J.Q. Xiao, J. S. Jiang, and C.L. Chien, , Phys. Rev. Lett. 68, 3749 (1992).

[3] M.N. Baibich, J.M. Broto, A. Fert, F. Nguyen Van Dau, and F. Petroff, P. Etienne, G. Creuzet, A. Friederich, and J. Chazelas, Phys. Rev. Lett., 61, 2472 (1988)

[4] G. Binasch, P. Grunberg, F. Saurenbach, and W. Zinn, Phys. Rev. B 39, 4828 (1989).

[5] R.E. Camley and J. Barnas, Phys. Rev. Lett. 63, 664 (1989).

[6] T. Valet, A. Fert, Phys. Rev. B 48, 7099 (1993).

[7] A. Vedyaev, B. Dieny, and N. Ryzhanova, Europhys. Lett., 19, 329 (1992).

[8] M. Rubinstein, Phys. Rev. B 50, 3830 (1994). and the moment-moment correlation function $C_{i j}=$ $\left\langle\boldsymbol{\mu}_{i} \cdot \boldsymbol{\mu}_{j}\right\rangle-\left\langle\boldsymbol{\mu}_{i}\right\rangle \cdot\left\langle\boldsymbol{\mu}_{j}\right\rangle:$

$$
\begin{aligned}
C_{i j} & =U_{i j}^{z z}\left(\frac{\partial \mathcal{L}(s)}{\partial s}\right)^{2}+\left(U_{i j}^{x x}+U_{i j}^{y y}\right)\left(\frac{\mathcal{L}(s)}{s}\right)^{2} \\
& =\frac{1-3 \cos ^{2} \theta_{i j}}{r_{i j}^{3}} \mathcal{L}_{2}(s)
\end{aligned}
$$

with $s=\mu_{0} h / k_{\mathrm{B}} T$. These expressions must still be averaged over the positions of the granules. We assume a uniform distribution with the excluded volume constraint [17] and obtain for $\left\langle\mu_{z}\right\rangle$ :

$$
\begin{aligned}
\left\langle\mu_{z}\right\rangle=\mathcal{L}(s)- & \beta \mu_{0}^{2} \mathcal{L}(s) \mathcal{L}^{\prime}(s) \times \\
& \times \frac{f}{V_{0}} \int_{\left|\mathbf{r}_{j}-\mathbf{r}_{i}\right|>2 r_{0}} d^{3} r_{j} U_{i j}^{z z},
\end{aligned}
$$

which can be written in terms of the classical demagnetizing factor $N_{z}$ of the granular sample as

$$
\left\langle\mu_{z}\right\rangle=\mathcal{L}(s)-f \frac{T_{0}}{T} \mathcal{L}(s) \mathcal{L}^{\prime}(s)\left(N_{z}-\frac{4 \pi}{3}\right) .
$$

Finally, the Fourier transform (restricted by the excluded volume) $C(\mathbf{q}) \equiv C_{\|}(\mathbf{q})+C_{\perp}(\mathbf{q})$ of the correlation function is

$$
\begin{aligned}
C(\mathbf{q}) & =\frac{f}{V_{0}} \int_{r>d} d^{3} \mathbf{r} C(\mathbf{r}) e^{i \mathbf{q} \cdot \mathbf{r}}= \\
& =\frac{8 \pi}{3} f \frac{T_{0}}{T} \mathcal{L}_{2}(s) \psi\left(2 q r_{0}\right) P_{2}\left(\cos \theta_{\mathbf{q}, \mathbf{h}}\right) .
\end{aligned}
$$

[9] Horacio E. Camblong, Peter M. Levy and Shufeng Zhang, Phys. Rev. B 51, 16052 (1995). Horacio E. Camblong, Shufeng Zhang and Peter M. Levy, J. Appl. Phys. 75, 6906 (1994).

[10] Peng Xiong, Gang Xiao, J. Q. Wang, John Q. Xiao, J. Samuel Jiang and C. L. Chien, Phys. Rev. Lett. 22, 3220 (1992). Jiang-Qing Wang and Gang Xiao, Phys Rev B 49, 3982 (1994).

[11] P. Allia, M. Knobel P. Tiberto and F. Vinai, Phys Rev B 52, 15398 (1995).

[12] S. Zhang and P. M. Levy, J. Appl. Phys. 73, 5315 (1994).

[13] Wendong Wang, Fengwu Zhu, Wuyan Lai, Jian-qing Wang, Gaiying Yang, Jing Zhu and Ze Zhang, J. Phys. D: Appl. Phys 32, 1990 (1999).

[14] E. F. Ferrari, F. C. S. da Silva and M. Knobel, Phys. Rev. B 56, 6086 (1997). 
[15] B. J. Hickey, M. A. Howson, S. O. Musa and N. Wiser, Phys. Rev B 51, 667 (1995).

[16] D. Altbir, P. Vargas, J. D'Albuquerque e Castro and U. Raff, Phys Rev B 57, 13604 (1998).

[17] Yu. G. Pogorelov, M. M. P. de Azevedo, and J. B. Sousa, Phys. Rev. B 58, 425 (1998) .

[18] M. El-Hilo, K. O' Grady and R. W. Chantrell, J. Appl. Phys. 76, 6811 (1994).

[19] D. Kechrakos and K.N. Trohidou, Phys. Rev. B 62, 3941 (2000).

[20] J.B. Sousa, M. M. Pereira de Azevedo, M. S. Rogalski, Yu. G. Pogorelov, L. M. Redondo, C. M. de Jesus, J. G. Marques, M. F. da Silva, J. C. Soares, J.-C. Ousset, and E. Snoeck, J. Mag. Mag. Materials, 196-197, 13 (1999).

[21] J. L. Gittleman, Y. Goldstein and Bozowski, Phys. Rev. B 5, 3609 (1972).

[22] D. Altbir, J. D' Albuquerque e Castro, P. Vargas, Phys. Rev. B 54 R6823 (1996).

[23] J. A. Mendes, V. S. Amaral, J. B. Sousa, L. Thomas and
B. Barbara, J. Appl. Phys. 81, 5208 (1997).

[24] Y. J. Chen, J. Ding, L. Si, W. Y. Cheung, S. P. Wong, L. H. Wilson, T. Suzuki, Appl. Phys. A 73, 103 (2001).

[25] J. M. V. Lopes, MSc thesis, Faculty of Sciences of the University of Porto (1999).

[26] J. M. V. Lopes, J.M.B. Lopes dos Santos, and Yu.G. Pogorelov, JMMM 242-245, 482 (2002).

[27] Ju.H. Kim, John Q. Xiao, C.L. Chien, and Z. Tesanovic, Solid State. Commun., 89, 157 (1994).

[28] A.A. Abrikosov, L.P. Gor'kov, and I.E. Dzyaloshinskii, Quantum Field Theoretical Methods in Statistical Physics, Oxford-N.Y., Pergamon Press, 1995.

[29] T. A. Rabedeau, M. F. Toney, R. F. Marks, S. S. P. Parkin, R. F. C. Parkin, R. F. C. Farrow and G. R. Harp, Phys Rev. B 48, 16810 (1993).

[30] Mary Beth Stearns and Yuanda Cheng, J. Appl. Phys. 75, 6894 (1994). 